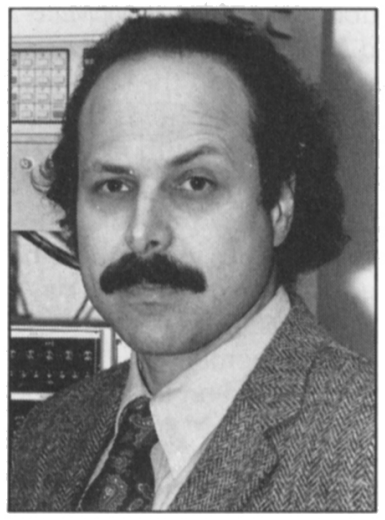

\section{Outstanding Young Investigator Award Goes to David Awschalom}

David D. Awschalom

David D. Awschalom, a professor in the Department of Physics at the University of California, Santa Barbara, is the 1992 recipient of the Materials Research Society's Outstanding Young Investigator Award.

Awschalom was nominated for his contributions to the physics of manmade materials. Among these contributions are the study and explanation of thermodynamic properties of molecular systems in confined geometries, the first direct observations of the dynamic and static magnetic properties in magnetic superlattices, and the first observation of macroscopic spin phenomena in nanometer-scale magnets.

Awschalom, who has been described by one of his nominators as "a popular speaker in various national and international meetings," will talk on "Spin Dynamics and Tunneling in Quantum Magnetic Systems" during Symposium X at the 1992 MRS Spring Meeting. The talk is scheduled for noon, Tuesday, April 28. His award is being presented during plenary and awards ceremonies scheduled for Monday evening, April 27.

Awschalom and co-workers brought a pioneering dimension to the study of the melting of molecular systems in porous glass, a relatively old subject, by preparing porous glass of extremely well-defined geometry using sol-gel techniques improved by thermal treatment. The resulting wellcharacterized glasses with a broad range of pore sizes and surface-area-to-volume ratios were used to demonstrate that the depression of the freezing point varied inversely in the pore radius.

"This experiment touched off a fury of experimental activities including works in the neutron and $x$-ray scattering, computer simulation, NMR, spin echo, thermodynamics, and light scattering communities," said Prof. Moses H.W. Chan of Pennsylvania State University, who first met Awscha- lom as a graduate student. "Awschalom's result is often cited as the reason for many subsequent papers in this area," he said.

Awschalom is also recognized for his development of time-resolved spectroscopy, which he has applied to many materials, such as the bulk magnetic semiconductor $\mathrm{Cd}_{1-x} \mathrm{Mn}_{x} \mathrm{Te}$, magnetic semiconductor superlattices (also based on the II-VI semiconductor class incorporating $\mathrm{Mn}$ ), and III-V superlattices. Awschalom and fellow

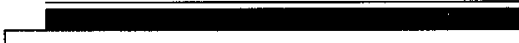 \\ MRS Outstanding Young Investigator Award \\ Purpose: To recognize outstanding, interdisciplinary scientific work in materials research by a young scien- tist or engineer. The awardee must also show exceptional promise as a developing leader in the materials area. \\ Nomination deadline for the 1993 Award is November 1, 1992. \\ For information about eligibility and nomination procedures, contact: Anne Wagner (OYI-B), Materials Research Society, 9800 McKnight Road, Pittsburgh, PA 15237; phone (412) 367-3003; fax (412) $367-4373$.}

researchers combined the most advanced aspects of SQUID technology with his own optical techniques to achieve sensitivities far exceeding those of resonance spectroscopies and to achieve time resolution of femtoseconds. These techniques have been applied to the formation of bound magnetic polarons in both bulk and confined geometries afforded by superlattices of different widths. "His work offers a complete description of the dynamics of polaron formation, spin flip processes of free carriers interacting with local $\mathrm{Mn}$ spins, and spin-spin exchange among carriers confined in quantum wells," said Stephan von Molnar of IBM's T.J. Watson Research Center.

Motivated by suggestions that "superparamagnetic" particles are likely to produce measurable manifestations of macroscopic quantum tunneling (MQT), Awschalom and colleague M.A. McCord developed methods for producing small ferromagnetic particles using a scanning tunneling microscope. Awschalom has used his miniature dc SQUID-based susceptometer to measure the frequency dependence of the magnetic susceptibility of arrays of such particles at low temperature ( $\mathrm{T}=25 \mathrm{mK}$ ). Although qualitatively suggestive of MQT, his results cannot be reconciled with present theoretical predictions. However, says Stephan von Molnar, "the work has generated much interest and activity in the condensed matter community." Awschalom recently reexamined these phenomena using samples of Ferritin extracted from horse spleen. This material consists of a particulariy uniform distribution of $\sim 100 \AA$ diameter spherical protein particles containing the iron compound. The new data and their interpretation confirm the MQT mechanism and are being written for publication.

Awschalom holds $\mathrm{PhD}$ and MSc degrees in physics from Cornell University and a BSc in physics from the University of Illinois-Urbana. Prior to joining the University of California, Awschalom held positions at the IBM T.J. Watson Research Center, Yorktown Heights, New York. 
Minerals \& Reactions at the Atomic ScaleHigh-Resolution TEM

Mineralogical Society

of America Short Course

Hueston Woods Lodge, Ohio

October 23-25, 1992

(before GSA Meeting)

\section{Organizer: Peter R. Buseck}

(Arizona State University)

The goals of the course are to (a) provide a background into the TEM as a mineralogical tool, (b) give an introduction to the principles underlying its operation, and (c) explore mineralogical applications and ways in which electron microscopy can augment our knowledge of mineral structures, chemistry, and origin. Special attention will be devoted to mineralogical applications. We expect to have a modern TEM for hands-on demonstration and exercises.

Topics and Speakers: general principles of transmission electron microscopy (P.Buseck); principles I: electron diffractionSAED \& CBED (J.Steeds); principles II: high-resolution image formation, simulation, and analysis (P. Self): inelastic interactionEDS chemical analysis (D.Peacor): EELS \& electron channeling (ALCHEMI) (P.Buseck); non-stoichiometry, polysomatism, and reactions in minerals (D. Veblen); polytypism \& stacking disorder (A.Baronnet); phase definition by HRTEM (F.Allen): diagenetic reactions \& processes: clays \& shales (D.Peacor): carbonates (R.Reeder); analysis of deformation in geological materials (D.Green): imaging transformation-induced microstructures (G.Nord).

for registration form, write or call the MSA office 113017 th Street NW

Suite 330

Washington, DC 20036 (202) $775-4344$

\section{9th International Field Emission Symposium}

International Field Emission Society

\section{Dalhousie University, Halifax, Nova Scotia, Canada August 10 - 14, 1992}

The 39th International Field Emission Symposium will deal with all topics related to the theory and application of field emission, field ionization, and field description. Contributions are solicited in the areas of field electron microscopy, field ion microscopy, scanning tunneling microscopy, field ionization mass spectroscopy, atom probe microanalysis and electron, ion, and liquid metal sources. A one-day symposium will deal with STM and AFM with special emphasis on nanostructure physics.

\section{Abstract Deadline: May 1, 1992}

The deadline for registration is July 1, 1992. For more information, contact:

H. Jurgen Kreuzer

Chairman, IFES ' 92

Department of Physics

Dalhousie University

Halifax, Nova Scotia, Canada B3H 315

Phone (902) 494-6594

Fax (902) 494-5191

E-Mail: Kreuzer@Dalac or Kreuzer@AC.DAL.CA

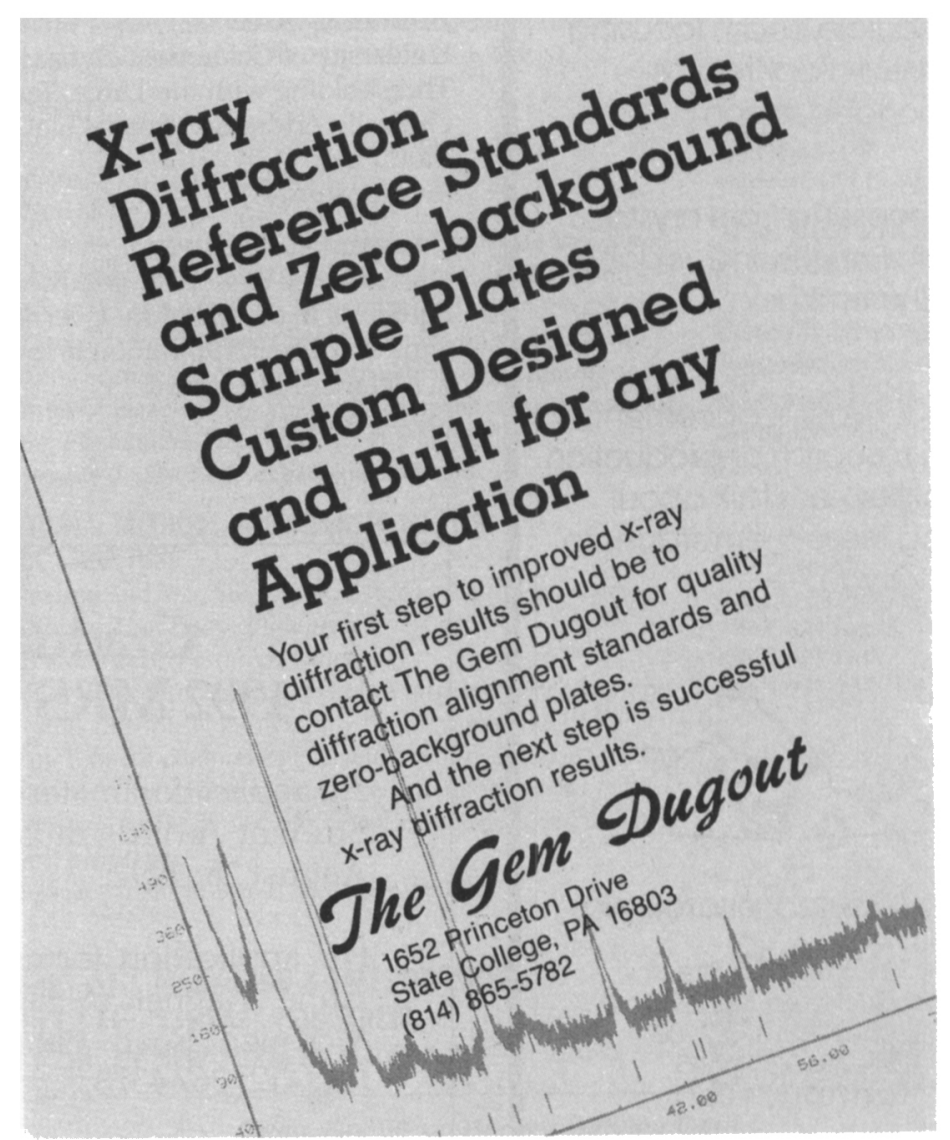

Circle No. 34 on Reader Service Card.

Circle No. 33 on Reader Service Card. 